\title{
Rabi oscillations in extreme ultraviolet ionization of atomic argon
}

\author{
Martin Flögel, Judith Durá, Bernd Schütte, Misha Ivanov, Arnaud Rouzée, and Marc J. J. Vrakking \\ Max-Born-Institut, Max-Born-Strasse 2A, 12489 Berlin, Germany
}

(Received 4 March 2016; published 10 February 2017)

\begin{abstract}
We demonstrate Rabi oscillations in nonlinear ionization of argon by an intense femtosecond extreme ultraviolet (XUV) laser field produced by high-harmonic generation. We monitor the formation of $\mathrm{Ar}^{2+}$ as a function of the time delay between the XUV pulse and an additional near-infrared (NIR) femtosecond laser pulse, and show that the population of an $\mathrm{Ar}^{+*}$ intermediate resonance exhibits strong modulations both due to an NIR laser-induced Stark shift and XUV-induced Rabi cycling between the ground state of $\mathrm{Ar}^{+}$and the $\mathrm{Ar}^{+*}$ excited state. Our experiment represents a direct experimental observation of a Rabi-cycling process in the XUV regime.
\end{abstract}

DOI: 10.1103/PhysRevA.95.021401

Intense coherent laser sources have enabled numerous techniques and applications that have transformed modern science. They have permitted the creation, control, and manipulation of new forms of matter by laser cooling $[1,2]$, the development of multiphoton microscopes with enhanced spatial resolution and molecular level selectivity [3], of optical standards achieving extraordinary precision based on the use of frequency combs [4], and have led to the generation of attosecond laser pulses, permitting time-resolved measurements at the electronic time scale [5]. So far, these developments are based on intense coherent laser sources in the infrared, visible, and ultraviolet, whereas until recently, no such sources have been available in the extreme ultraviolet (XUV, 10-124 nm). However, this situation has changed with the development of XUV or X-ray free-electron lasers (FELs) and intense coherent table-top XUV sources based on high-harmonic generation (HHG).

At FELs such as FLASH [6] and FERMI [7], intense XUV pulses are available with energies of a few hundreds of $\mu \mathrm{J}$, femtosecond-domain pulse durations and partial temporal coherence, unless coherent seeding (e.g., from an HHG source) is used. FELs have already successfully been used in pioneering experiments on multiphoton ionization [8] and coherent diffractive imaging of nanostructures [9]. HHG sources are a laboratory-scale alternative, with the advantage of full temporal coherence and synchronization to the driver laser, and hence to other derived sources accessing the ultraviolet, visible, infrared, and terahertz domains. HHG provides lower pulse energies than FELs; however, with available $\mu \mathrm{J}$-level pulse energies $[10,11]$ nonlinear XUV-induced processes have been observed in Refs. [12,13].

Rabi oscillations are one of the most fundamental manifestations of coherent light-matter interactions [14]. In a resonantly excited two-state quantum system, the state populations oscillate with a period $T=2 \pi / \Omega_{R}$, where the Rabi frequency $\Omega_{R}=\mu F / \hbar$, with $\mu$ the transition dipole moment and $F$ the electric field strength [15]. Rabi oscillations have been used in coherent control protocols [16] in atomic clocks [17] and in studies on quantum information [18].

Rabi cycling has been directly observed under the influence of low-frequency laser fields up to the ultraviolet [16-20], whereas at higher photon energies only indirect signatures have been found. For instance, Sato et al. [21] found deviations

*Corresponding author: judith.dura@mbi-berlin.de from lowest-order perturbation theory in ionization of He using the SPring-8 Compact SASE Source, which were attributed to Rabi oscillations [22]. Rabi cycling in x-ray-induced resonant Auger excitation of $\mathrm{Ne}$ was theoretically proposed [23] and inferred in experiments realized at the Linac Coherent Light Source [24].

In this Rapid Communication, we report Rabi oscillations during double ionization of Ar by intense XUV radiation obtained by HHG. A first XUV photoabsorption ionizes Ar and the XUV couples the produced $\mathrm{Ar}^{+}$to one of its excited states, $\mathrm{Ar}^{+*}$. Rabi oscillations are observed by ionizing the $\mathrm{Ar}^{+*}$ at a variable time delay using an NIR pulse, thus forming $\mathrm{Ar}^{2+}$. The XUV-NIR delay-dependent measurements moreover reveal an NIR laser-induced Stark effect.

A description of the experimental setup is given in [25]. Briefly, a $50 \mathrm{~Hz}, 35 \mathrm{~mJ}, 35 \mathrm{fs}$ Ti:sapphire laser $(\lambda=790 \mathrm{~nm})$ was split into two beams before compression. $80 \%$ of the output was used in a long focal length $(5 \mathrm{~m})$ HHG beamline, using a 15-cm-long gas cell statically filled with 1.3 mbar Xe. After propagation, the remaining NIR field was blocked using a $100 \mathrm{~nm}$ aluminum filter. The second NIR beam was independently compressed and recombined with the XUV using a holey mirror $(\varnothing=6 \mathrm{~mm})$. Both the XUV and NIR beams were focused at the center of a velocity map imaging spectrometer (VMI) [26] using a spherical $\mathrm{B}_{4} \mathrm{C}$-coated mirror (75 mm focal length), which selected harmonics H11-H17 of the XUV beam $(h v=17.32,20.48,23.62$, and $26.78 \mathrm{eV})$. The XUV pulse energy in the experiment was up to $10 \mathrm{~nJ}$, suggesting an intensity of $3.5 \times 10^{12} \mathrm{~W} / \mathrm{cm}^{2}$ for an estimated focal radius of $3 \mu \mathrm{m}$ [25]. Using terahertz streaking the typical XUV pulse duration was previously measured [27] to be $20 \pm 3$ fs, subject to day-to-day variations. The NIR peak intensity was calibrated using the ratio of the $\mathrm{Ar}^{+}$and $\mathrm{Ar}^{2+}$ ionization yields [28]. To avoid significant NIR-only formation of $\mathrm{Ar}^{+}$and $\mathrm{Ar}^{2+}$, the NIR peak intensity was varied during the experiment between $7.5 \times 10^{12} \mathrm{~W} / \mathrm{cm}^{2}$ and $5 \times 10^{13} \mathrm{~W} / \mathrm{cm}^{2}$, using a half-wave plate and a polarizer. The zero XUV-NIR delay and the NIR pulse duration (71 \pm 3 fs FWHM) were determined by a "sideband" measurement [29]. This NIR pulse duration exceeds the aforementioned value due to dispersion along the beam path. The NIR focal radius was estimated to be $\approx 12 \mu \mathrm{m}$. At the focus, both laser beams crossed an Ar beam formed by expanding 1 bar of Ar into vacuum using a piezoelectric valve with a $500 \mu \mathrm{m}$ nozzle diameter. Two-dimensional (2D) VMI projections of the ion and electron 


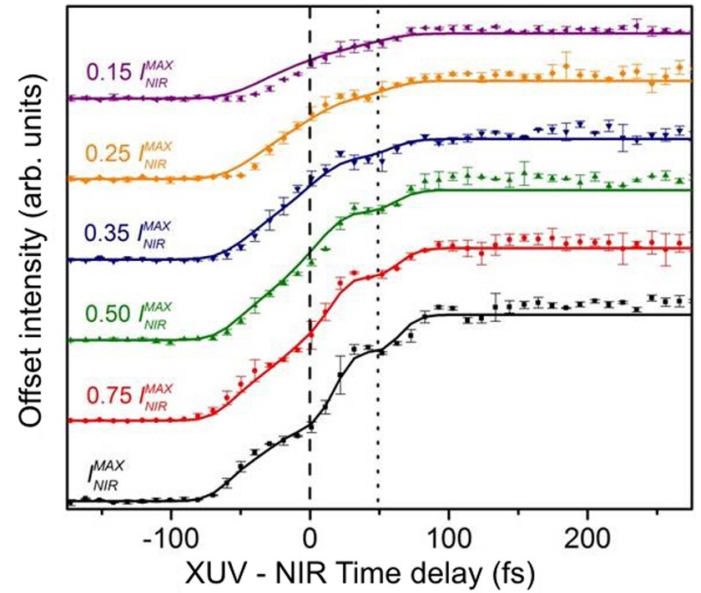

FIG. 1. $\mathrm{Ar}^{2+}$ ion yield as a function of the XUV pump-NIR probe time delay for different NIR intensities. The experimental yields (symbols) were extracted by integrating $\mathrm{Ar}^{2+}$ signals over 256 laser shots. The error bars correspond to the standard deviation of two independent measurements. Different symbols correspond to different intensities indicated on the left side as a fraction of $I_{\mathrm{NIR}}^{\mathrm{MAX}}$. Numerical modeling yields $I_{\mathrm{NIR}}^{\mathrm{MAX}}=2.0 \times 10^{12} \mathrm{~W} / \mathrm{cm}^{2}$. The experimental data is fitted (continuous lines) using the expression given in Eq. (4) for $t \rightarrow \infty$. Vertical black lines at 0 and $50 \mathrm{fs}$ time delay indicate modulations in the $\mathrm{Ar}^{2+}$ ion yield explained in the text.

momentum distributions were recorded using a microchannel plate and phosphor screen assembly and a CCD camera. Abel inversion of the $2 \mathrm{D}$ projections allowed retrieval of the kinetic energy distributions [30].

Experimentally, the dependence of the $\mathrm{Ar}^{2+}$ yield on the XUV-NIR time delay was recorded. The $\mathrm{Ar}^{2+}$ ion yield is presented in Fig. 1 for six different NIR intensities ranging from $3 \times 10^{11} \mathrm{~W} / \mathrm{cm}^{2}$ to $I_{\mathrm{NIR}}^{\mathrm{MAX}}=2.0 \times 10^{12} \mathrm{~W} / \mathrm{cm}^{2}$ (intensities based on modeling, to be discussed below). For large negative delays (NIR before XUV) the $\mathrm{Ar}^{2+}$ yield equals the XUV-only yield. For large positive delays (NIR after XUV), a strongly enhanced $\mathrm{Ar}^{2+}$ yield is observed. Surprisingly, the rise of the $\mathrm{Ar}^{2+}$ yield with XUV-NIR delay shows pronounced modulations at 0 and 50 fs delay (dashed and dotted lines in Fig. 1). While the modulation near 0 fs vanishes with decreasing NIR intensity, the modulation at 50 fs remains visible over the whole NIR intensity range.

In Fig. 2(a), the $\mathrm{Ar}^{+}$and $\mathrm{Ar}^{2+}$ yields are displayed as a function of the XUV-NIR time delay for $I_{\mathrm{NIR}}^{\mathrm{MAX}}$, together with their sum. The $\mathrm{Ar}^{2+}$ increase with time delay is accompanied by a decrease of the $\mathrm{Ar}^{+}$yield. Since NIR ionization of the $\mathrm{Ar}^{+}$ground state is negligible at the NIR intensities used, we conclude that the $\mathrm{Ar}^{2+}$ formation proceeds via the ionization of excited $\mathrm{Ar}^{+*}$. Figure 2(b) shows the $\mathrm{Ar}^{2+}$ yield dependence on the XUV intensity at $I_{\mathrm{NIR}}^{\mathrm{MAX}}$ for a large positive delay of $300 \mathrm{fs}$, obtained by measuring the $\mathrm{Ar}^{2+}$ yield as a function of the $\mathrm{Ar}^{+}$yield, which depends linearly on the XUV intensity. The $\mathrm{Ar}^{2+}$ yield follows a near-quadratic power law with respect to the XUV intensity, $I_{\mathrm{XUV}}$, i.e., $S_{\mathrm{Ar}^{2+}}(t)=\sigma I_{\mathrm{XUV}}^{2}$, with $\sigma$ the effective two-photon ionization cross section. We note that for long time delays the $\mathrm{Ar}^{2+}$ yield varies linearly with the NIR intensity, suggesting that predominantly a single NIR photon is involved in the ionization process.

Electron VMI measurements were performed for a large positive delay ( $300 \mathrm{fs}$ ) between the XUV and the NIR pulses at $I_{\mathrm{NIR}}^{\mathrm{MAX}}$, and for the XUV pulse only. A slice through the difference between these two measurements is shown as the inset in Fig. 3(a), together with the corresponding photoelectron kinetic energy distribution. The NIR laser introduces new contributions in the photoelectron spectrum at $0.13,0.75$, and $2.35 \mathrm{eV}$. Moreover, near-zero kinetic energy electrons are observed from two-color $\mathrm{XUV}+\mathrm{NIR}$ ionization of Ar clusters in the beam, as previously reported in [13]. We assign the $0.75 \mathrm{eV}$ photoelectrons to NIR single-photon ionization of $\mathrm{Ar}^{+*}$ ions that are prepared, starting from the neutral Ar ground state, by the absorption of two XUV photons. We propose that an excited $\mathrm{Ar}^{+*}$ ion is formed by absorption of one photon of $\mathrm{H} 17(h v=26.78 \mathrm{eV})$ by a ground state $\mathrm{Ar}^{+} 3 p^{5}\left({ }^{2} P_{1 / 2,3 / 2}\right)$ ion, that itself is formed by absorption of one of the harmonics $\mathrm{H} 11-\mathrm{H} 17$ by a neutral $\operatorname{Ar} 3 p^{6}\left({ }^{1} S\right)$ atom [see Fig. 3(b)]. Within the bandwidth of H17, five excited $\mathrm{Ar}^{+}$states are reachable via dipole-allowed transitions from the $\operatorname{Ar}^{+} 3 p^{5}\left({ }^{2} P_{1 / 2,3 / 2}\right)$ ground states [31], namely, $3 s^{2} 3 p^{4}\left({ }^{1} D\right) 6 s\left({ }^{2} D_{3 / 2,5 / 2}\right), 3 s^{2} 3 p^{4}\left({ }^{1} D\right) 5 d\left({ }^{2} D_{5 / 2}\right), 3 s^{2} 3 p^{4}\left({ }^{1} S\right)$ $5 s\left({ }^{2} S_{1 / 2}\right)$, and $3 s^{2} 3 p^{4}\left({ }^{1} D\right) 5 d\left({ }^{2} S_{1 / 2}\right)$ [31]. The latter state has the highest oscillator strength [32,33], namely, two to four times larger than the others, and the highest cross section for NIR ionization [32]. The photoelectron peak near $2.35 \mathrm{eV}$ originates from two-photon NIR ionization of $\mathrm{Ar}^{+*}$. To date, we have not been able to assign the small peak at $0.13 \mathrm{eV}$ to a contribution from either Ar atoms or Ar clusters.

To understand the $\mathrm{Ar}^{2+}$ ion yield dependence on the XUV-NIR time delay, we use a quantum-mechanical model with the following simplifying assumptions: (i) $\mathrm{The}^{+} \mathrm{Ar}^{+}$ion is considered as the ground state, i.e., the first ionization step, $\operatorname{Ar} 3 p^{6}\left({ }^{1} S\right)+1 h v_{\mathrm{XUV}} \rightarrow \operatorname{Ar}^{+} 3 p^{5}\left({ }^{2} P_{1 / 2,2 \beta}\right)+e_{1}^{-}$, is not included. (ii) $\mathrm{The}^{+} \mathrm{Ar}^{+}$ion is regarded as a two-level system coupled by the H17 XUV laser field, where the initial energy $E_{0}$ is given by the $\operatorname{Ar}^{+} 3 p^{5}\left({ }^{2} P_{3 / 2}\right)$ ground state and the excited state energy $E_{1}$ by that of the $\mathrm{Ar}^{+*}$ excited state, chosen for simplicity as an effective state with an energy calculated by taking the weighted average of the energies of the aforementioned five possible $\mathrm{Ar}^{+*}$ states, with the square of the transition dipole moments as weights. The calculation of the effective transition dipole moment is described in the Supplemental Material [34]. (iii) In the probe step, a single NIR photon ionizes the $\mathrm{Ar}^{+*}$, i.e., $\mathrm{Ar}^{+*}+1 h v_{\mathrm{NIR}} \rightarrow$ $\operatorname{Ar}^{2+} 3 p^{4}\left({ }^{3} P_{0,1,2}\right)+e_{2}^{-}$. Under these assumptions, the total wave function $\Psi(t)$ can be written as a superposition of the $\mathrm{Ar}^{+}$ground state $\left(\varphi_{0}\right)$, the $\mathrm{Ar}^{+*}$ excited state $\left(\varphi_{1}\right)$, and the $\mathrm{Ar}^{2+}+e_{2}^{-}$continuum $\left(\varphi_{E}\right)$ as [35]

$$
\begin{aligned}
\Psi(t)= & C_{0}(t) \varphi_{0} e^{-(i \hbar) E_{0} t}+C_{1}(t) \varphi_{1} e^{-(i \hbar) E_{1} t} \\
& +\int_{0}^{\infty} d E C_{E}(t) \varphi_{E} e^{-(i \hbar) E t},
\end{aligned}
$$

where $E$ is the energy of the continuum state (i.e., the sum of the $\mathrm{Ar}^{2+}$ energy and the photoelectron energy) and $\left|C_{0,1, E}(t)\right|^{2}$ are the populations of the ground, excited, and final states as a function of time. The $C_{0,1, E}(t)$ are determined by solving the time-dependent Schrödinger equation $[\hat{H}+\hat{V}(t)] \Psi(t)=$ 

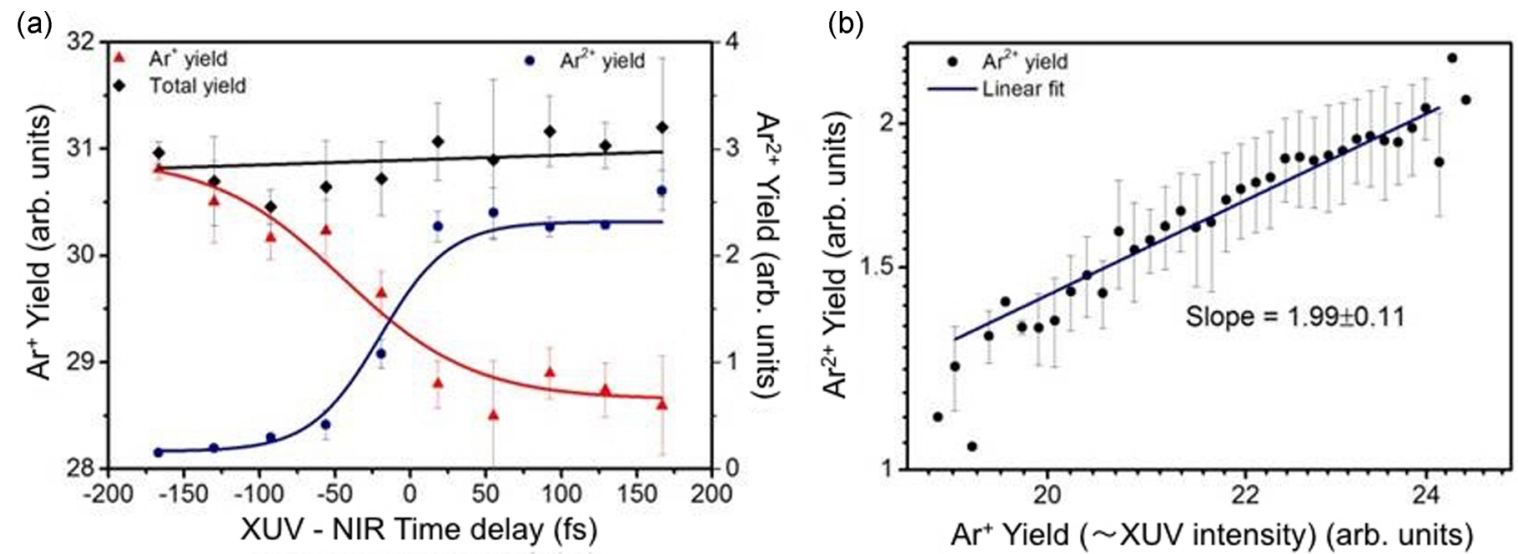

FIG. 2. (a) Experimental $\mathrm{Ar}^{2+}$ (blue dots) and $\mathrm{Ar}^{+}$yield (red triangles), and their sum (black diamonds) as a function of the XUV-NIR time delay for $I_{\mathrm{NIR}}^{\mathrm{MAX}}$. Continuous lines are a linear fit of the summed yield (black) and sigmoidal fits of the $\mathrm{Ar}^{2+}$ and $\mathrm{Ar}^{+}$yields (blue and red), respectively. The ionic yields were extracted by integrating over 256 laser shots. The error bars correspond to the standard deviation of nine independent measurements. (b) Log-log plot of the $\mathrm{Ar}^{2+}$ yield as a function of the $\mathrm{Ar}^{+}$yield, obtained by recording 1000 single-shot time-of-flight spectra at a $300 \mathrm{fs}$ XUV-NIR time delay, for $I_{\mathrm{NIR}}^{\mathrm{MAX}}$. The dots are the mean of binned experimental data and the error bars correspond to the standard deviation. The linear fit (continuous blue line) gives a slope of $1.99 \pm 0.11$, and illustrates that the $\mathrm{Ar}^{2+}$ yield depends quadratically on the XUV intensity.

$i \hbar(\partial / \partial t) \Psi(t)$, where $\hat{H}$ is the Hamilton operator of the unperturbed system and $\hat{V}(t)$ represents the atom-laser interaction, with $\hat{V}(t)=\sum_{j} \hat{\mu} f_{j}(t) F_{j} \cos \left(\omega_{j} t\right)$. Here, $\hat{\mu}$ is the dipole operator, $f_{j}(t)$ is the field envelope (with $\cos ^{2}$ pulses assumed), $F_{j}$ is the maximum field strength, and $\omega_{j}$ is the angular frequency of the $j=\mathrm{XUV}$ or NIR field, respectively. Using the standard approach described, for example, in [35], one obtains

$$
\begin{gathered}
i \hbar \dot{C}_{0}(t)=-\frac{1}{2} \mu_{01} F_{\mathrm{XUV}} f_{\mathrm{XUV}}(t) C_{1}(t), \\
i \hbar \dot{C}_{1}(t)=-\frac{1}{2} \mu_{10} F_{\mathrm{XUV}} f_{\mathrm{XUV}}(t) C_{0}(t)-\Delta(t) C_{1}(t) \\
-i \pi f_{\mathrm{NIR}}^{2}(t)\left|V_{1, E_{1}+\hbar \omega_{\mathrm{NIR}}}\right|^{2} C_{1}(t) .
\end{gathered}
$$

Here, $\Delta(t)$ incorporates the Stark shift of the $\mathrm{Ar}^{+*}$ state, $\Delta(t)=\Delta_{e}-U_{p}(t)$, where $\Delta_{e}=E_{0}-E_{1}+\hbar \omega_{\mathrm{XUV}}$ is the XUV detuning and $U_{p}(t)$ is the ponderomotive energy of the NIR field $\left(U_{p}(t)=e^{2} F_{\mathrm{NIR}}(t)^{2} / 4 m \omega_{\mathrm{NIR}}^{2}\right.$, with $e$ the electron charge and $m$ the electron mass).

The $\mathrm{Ar}^{2+}$ yield can be expressed as

$$
S_{\mathrm{Ar}^{2+}}(t)=\left.\frac{2 \pi}{\hbar}\left|V_{1, E}\right|^{2}\right|_{E=E_{1}+\hbar \omega_{\mathrm{NIR}}} \int_{-\infty}^{t} d t^{\prime}\left|C_{1}\left(t^{\prime}\right)\right|^{2} f_{\mathrm{NIR}}^{2}\left(t^{\prime}\right) .
$$

The measured $\mathrm{Ar}^{2+}$ yields correspond to taking the limit $t \rightarrow \infty$ of this equation. Figure 1 shows simulated $\mathrm{Ar}^{2+}$ yields as a function of the XUV-NIR delay, obtained by globally fitting the experimental $\mathrm{Ar}^{2+}$ XUV-NIR time delay

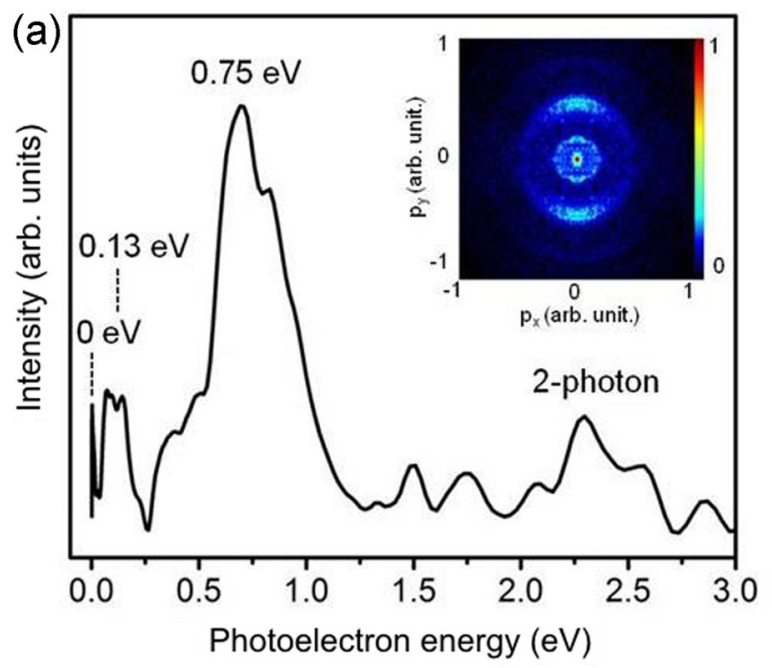

(b)

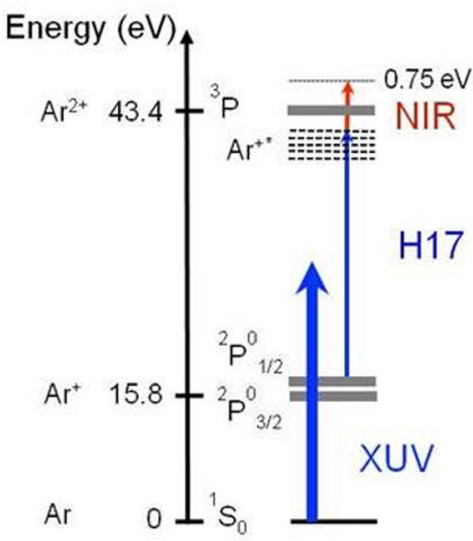

FIG. 3. (a) Angle-integrated low kinetic energy photoelectron spectrum for ionization of Ar atoms with an XUV pump and an NIR probe delayed by $300 \mathrm{fs}$, derived from the normalized differential two-color 2D photoelectron momentum distribution shown in the inset. (b) Energy diagram (not scaled), depicting double ionization of Ar by two XUV photons, where the second XUV photon is H17, and one NIR photon. Excited ions, $\mathrm{Ar}^{+*}$, prepared by absorption of the H17 photon, are ionized by the NIR photon. 


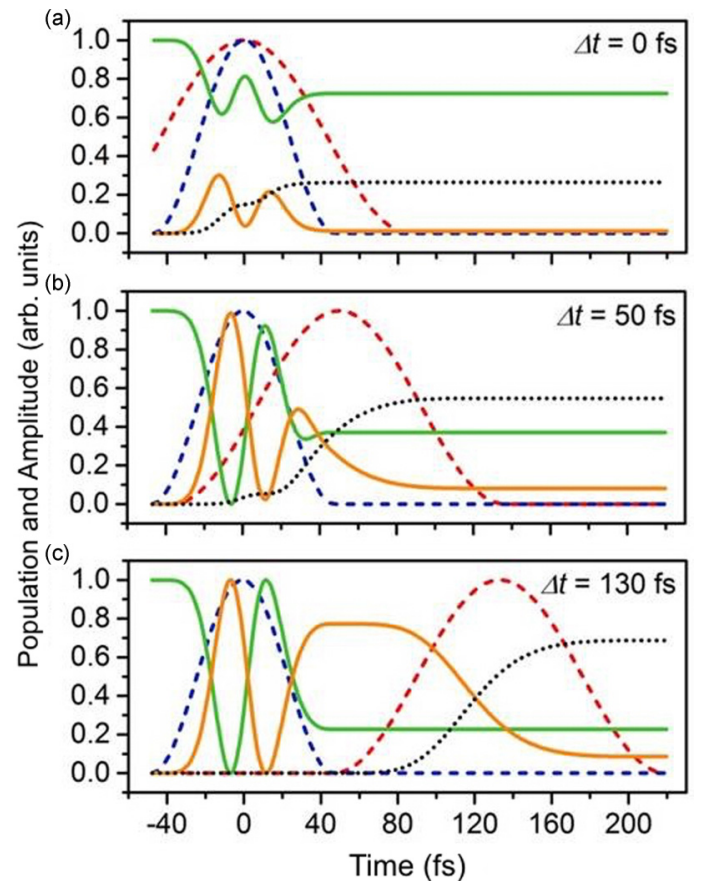

FIG. 4. Populations, $\left|C_{0}(t)\right|^{2}$ (continuous green line) and $\left|C_{1}(t)\right|^{2}$ (continuous orange line), of the $\mathrm{Ar}^{+}$ground and excited states, respectively, along with the relative field envelopes, $f_{\mathrm{NIR}}(t)$ (dashed red line) and $f_{\mathrm{XUV}}(t)$ (dashed blue line) and the time-integrated ionization probability $S_{\mathrm{Ar}^{2+}}(t)$ (dotted black line), for $I_{\mathrm{NIR}}^{\mathrm{MAX}}$, at two XUV-NIR pulses delay times: (a) $0 \mathrm{fs}$, (b) $50 \mathrm{fs}$, and (c) $130 \mathrm{fs}$.

dependence using Eq. (4), for the indicated NIR intensities (continuous lines). The model very well reproduces the experimental $\mathrm{Ar}^{2+}$ yields including the aforementioned modulations at 0 and $50 \mathrm{fs}$. The physical quantities used in our model as fitting parameters are described in the Supplemental Material [34]. The fit returned $I_{\mathrm{XUV}}=3.5 \times 10^{12} \mathrm{~W} / \mathrm{cm}^{2}$ with a pulse duration $\tau_{\mathrm{XUV}}=47.4 \pm 0.2 \mathrm{fs}(\mathrm{FWHM})$, and $I_{\mathrm{NIR}}^{\mathrm{MAX}}=$ $2.0 \times 10^{12} \mathrm{~W} / \mathrm{cm}^{2}$ with a pulse duration $\tau_{\mathrm{NIR}}=86.2 \pm 2.0 \mathrm{fs}$ (FWHM). All these values are close to the experimentally expected ones, with the exception of the NIR peak intensity. However, a lower-than-expected NIR peak intensity can easily be explained by a transverse or longitudinal displacement of the NIR focus. Independent confirmation of the returned NIR intensity is the ability to unambiguously identify the $\mathrm{Ar}^{+*}$ excited state in the photoelectron spectrum. Moreover, for NIR intensities of $5 \times 10^{13} \mathrm{~W} / \mathrm{cm}^{2}\left(U_{p}=2.92 \mathrm{eV}\right)$, as were expected experimentally, the $\mathrm{Ar}^{+*}$ state would completely move out of resonance with $\mathrm{H} 17$.

Insight into the processes responsible for the modulations near 0 and 50 fs time delay is obtained by plotting the time-dependent population of the ground and excited states according to Eq. (4). Figure 4 displays $\left|C_{0}(t)\right|^{2}$ and $\left|C_{1}(t)\right|^{2}$ for 0 and 50 fs time delay, together with the time-integrated ionization probability $S_{\mathrm{Ar}^{2+}}(t)$, for the parameters determined from the fit. The relative XUV and NIR field envelopes are also displayed. When the two pulses overlap in time $[\Delta t=0 \mathrm{fs}$, Fig. 4(a)], a depletion of the maximum population of the excited state $\left(\left|C_{1}(t)\right|^{2}\right.$, orange line) is observed together with a minor decrease of the $\mathrm{Ar}^{+}$ground state population $\left(\left|C_{0}(t)\right|^{2}\right.$, green line). Since the XUV field is resonant with the effective state, $E_{0}-E_{1}+\hbar \omega_{\mathrm{XUV}} \approx 0$, a local minimum in the total yield of $\mathrm{Ar}^{2+}$ in Fig. 1 occurs at $\Delta t=0 \mathrm{fs}$ when the ac Stark shift induced by the NIR field becomes comparable to the bandwidth of $\mathrm{H} 17(\sim 0.25-0.3 \mathrm{eV})$, i.e., for. $I_{\mathrm{NIR}}>0.5 I_{\mathrm{NIR}}^{\mathrm{MAX}}$.

Figures $4(\mathrm{~b})$ and $4(\mathrm{c})$ display $\left|C_{0}(t)\right|^{2}$ and $\left|C_{1}(t)\right|^{2}$ for a time delay of 50 and $130 \mathrm{fs}$, respectively, between the XUV and NIR laser pulses. Both populations oscillate out of phase with each other, with a modulation depth of almost 1 . This modulation is due to Rabi cycling between the ground and excited ionic states with a corresponding Rabi frequency $\Omega_{R}=0.174 \mathrm{fs}^{-1}$ for $I_{\mathrm{XUV}}=3.5 \times 10^{12} \mathrm{~W} / \mathrm{cm}^{2}$. At $50 \mathrm{fs}$ pump-probe time delay the two pulses are sufficiently far apart that the NIR laser will not influence anymore the XUV-driven transition to the excited state. The Rabi-cycling process driven by the XUV field leads to a minimum of population transferred to the excited state at a time of $50 \mathrm{fs}$, i.e., when the IR field arrives, as shown in Fig. 4(b). At $\Delta t=130 \mathrm{fs}$ in Fig. 4(c), the population of the excited state has a final amplitude of 0.75. The NIR field is ionizing most of the population of the excited state with a $S_{\mathrm{Ar}^{2+}}(t)$ amplitude of 0.65. In Fig. 1, the influence of the Rabi oscillation at $\Delta t=50 \mathrm{fs}$ on the integrated ionization probability led to the observation of a single local minimum in the measured $\mathrm{Ar}^{2+}$ yield at an XUV-NIR delay of 50 fs (dotted black vertical line). Since $\Omega_{R}=\mu F / \hbar$, the Rabi oscillations are strongly influenced by the XUV intensity. Our experimental observation is the result of a weighted focal volume averaging over the spatial distribution of XUV intensities in the interaction volume. However, in this weighting higher intensities are favored since the formation of the $\mathrm{Ar}^{+}$ground state already relies on the absorption of an XUV photon. Volume averaging over the NIR laser focus is avoided [36] since the NIR beam has a significantly larger focal radius size $(\approx 12 \mu \mathrm{m})$ than the XUV beam $(\approx 3 \mu \mathrm{m})$. The limited influence of the NIR intensity on the visibility of the Rabi oscillations for longer time delays is exemplified by the persistence of the local minimum at 50 fs time delay in Fig. 1 when the NIR intensity is varied.

In conclusion, we have presented a direct experimental observation of a Rabi-cycling process in the XUV regime. By monitoring the $\mathrm{Ar}^{2+}$ yield as a function of XUV-NIR time delay, we have demonstrated an ionization mechanism where two XUV photons are sequentially absorbed, with a first XUV photon singly ionizing neutral Ar atoms and the second XUV photon resonantly coupling the $\operatorname{Ar}^{+} 3 p^{5}\left({ }^{2} P_{1 / 2,3 / 2}\right)$ ground state with an excited ionic state, $\mathrm{Ar}^{+*}$. $\mathrm{The} \mathrm{Ar}^{+*}$ population exhibits strong modulations due to an NIR-induced Stark effect and a Rabi-cycling process with the $\mathrm{Ar}^{+}$ground state. These experiments pave the way for the development of efficient coherent control techniques in multiphoton processes with laser frequencies in the XUV regime.

The authors wish to thank Professor Hugo van der Hart (Queen's University, Belfast) for useful discussions.

M.F. and J.D. contributed equally to this work. 
[1] M. H. Anderson, J. R. Ensher, M. R. Matthews, C. E. Wieman, and E. A. Cornell, Science 269, 198 (1995).

[2] M. E. J. Friese, T. A. Nieminen, N. R. Heckenberg, and H. Rubinsztein-Dunlop, Nature (London) 394, 348 (1998).

[3] T. A. Klar, S. Jakobs, M. Dyba, A. Egner, and S. W. Hell, Proc. Natl. Acad. Sci. 97, 8206 (2000).

[4] T. Udem, R. Holzwarth, and T. W. Hänsch, Nature (London) 416, 233 (2002).

[5] F. Krausz and M. Ivanov, Rev. Mod. Phys. 81, 163 (2009).

[6] W. Ackermann et al., Nat. Photonics 1, 336 (2007).

[7] E. Allaria et al., Nat. Photonics 6, 699 (2012).

[8] A. A. Sorokin, S. V. Bobashev, T. Feigl, K. Tiedtke, H. Wabnitz, and M. Richter, Phys. Rev. Lett. 99, 213002 (2007).

[9] H. N. Chapman et al., Nat. Phys. 2, 839 (2006).

[10] J. F. Hergott, M. Kovacev, H. Merdji, C. Hubert, Y. Mairesse, E. Jean, P. Breger, P. Agostini, B. Carré, and P. Salières, Phys. Rev. A 66, 021801(R) (2002).

[11] E. Takahashi, Y. Nabekawa, T. Otsuka, M. Obara, and K. Midorikawa, Phys. Rev. A 66, 021802(R) (2002).

[12] Y. Nabekawa, H. Hasegawa, E. J. Takahashi, and K. Midorikawa, Phys. Rev. Lett. 94, 043001 (2005).

[13] B. Schütte, M. Arbeiter, T. Fennel, M. J. J. Vrakking, and A. Rouzèe, Phys. Rev. Lett. 112, 073003 (2014).

[14] I. I. Rabi, Phys. Rev. 51, 652 (1937).

[15] F. H. M. Faisal, Theory of Multiphoton Processes (Springer Science + Business Media, New York, 1987).

[16] M. Yan, B. J. DeSalvo, Y. Huang, P. Naidon, and T. C. Killian, Phys. Rev. Lett. 111, 150402 (2013).

[17] P. J. Windpassinger, D. Oblak, P. G. Petrov, M. Kubasik, M. Saffman, C. L. Garrido-Alzar, J. Appel, J. H. Müller, N. Kjærgaard, and E. S. Polzik, Phys. Rev. Lett. 100, 103601 (2008).

[18] E. D. Kim, K. Truex, Y. Wu, A. Amo, X. Xu, D. G. Steel, A. S. Bracker, D. Gammon, and L. J. Sham, Appl. Phys. Lett. 97, 113110-3 (2010).

[19] M. Fushitani, C. N. Liu, A. Matsuda, T. Endo, Y. Toida, M. Nagasono, T. Togashi, M. Yabashi, T. Ishikawa, Y. Hikosaka et al., Nat. Photonics 10, 102 (2016).

[20] Q. Liao, Y. Zhou, C. Huang, and P. Lu, New J. Phys. 14, 013001 (2012).

[21] T. Sato et al., J. Phys. B 44, 161001 (2011).

[22] T. Sako, J. Adachi, A. Yagishita, M. Yabashi, T. Tanaka, M. Nagasono, and T. Ishikawa, Phys. Rev. A 84, 053419 (2011).
[23] N. Rohringer and R. Santra, Phys. Rev. A 77, 053404 (2008).

[24] E. P. Kanter, B. Krässig, Y. Li, A. M. March, P. Ho, N. Rohringer, R. Santra, S. H. Southworth, L. F. DiMauro, G. Doumy, C. A. Roedig, N. Berrah, L. Fang, M. Hoener, P. H. Bucksbaum, S. Ghimire, D. A. Reis, J. D. Bozek, C. Bostedt, M. Messerschmidt, and L. Young, Phys. Rev. Lett. 107, 233001 (2011).

[25] B. Schütte, F. Campi, M. Arbeiter, T. Fennel, M. J. J. Vrakking, and A. Rouzée, Phys. Rev. Lett. 112, 253401 (2014).

[26] A. T. J. B. Eppink and D. H. Parker, Rev. Sci. Instrum. 68, 3477 (1998).

[27] B. Schütte, T. Oelze, M. Krikunova, M. Arbeiter, T. Fennel, M. J. J. Vrakking, and A. Rouzée, New J. Phys. 17, 033043 (2015).

[28] A. Becker and F. H. M. Faisal, J. Phys. B 32, L335 (1999).

[29] T. E. Glover, R. W. Schoenlein, A. H. Chin, and C. V. Shank, Phys. Rev. Lett. 76, 2468 (1996).

[30] B. J. Whitaker, Image Reconstruction: The Abel Transform in Imaging in Chemical Dynamics, in ACS Symposium Series Vol. 770, edited by A. G. Suits and R. E. Continetti (American Chemical Society, Washington, DC, 2000).

[31] NIST Atomic Spectra Database, http://physics.nist.gov/cgibin/ASD/energy1.pl.

[32] The Opacity Project, http://cdsweb.u-strasbg.fr/topbase/ fvalues.html.

[33] A. R. Edmonds, Angular Momentum in Quantum Mechanics (Princeton University Press, Princeton, NJ, 1957).

[34] See Supplemental Material at http://link.aps.org/supplemental/ 10.1103/PhysRevA.95.021401 for the following. A description of the calculation of the transition dipole moment from the ionic argon ground state $\left(\mathrm{Ar}^{+}\right)$to an effective ionic excited state $\left(\mathrm{Ar}^{+*}\right)$, based on the relation between the transition dipole moment and the oscillator strength of the transition; some calculated oscillator strengths are compared with literature values showing a good agreement between them (Sec. 1). Also, a description of the physical quantities used in the quantum mechanical model applied in the Letter to fit the experimental data, i.e., the $\mathrm{Ar}^{2+}$ ionization yield as a function of the XUV-NIR time delay for different NIR intensities (Sec. 2).

[35] M. V. Fedorov and A. E. Kazakov, Prog. Quantum Electron. 13, 1 (1989).

[36] B. Schütte, M. Arbeiter, A. Mermillod-Blondin, M. J. J. Vrakking, A. Rouzée, and T. Fennel, Phys. Rev. Lett. 116, 033001 (2016). 